\title{
Jamming with Tunable Roughness
}

\author{
Harukuni Ikeda $\odot,{ }^{1, *}$ Carolina Brito, ${ }^{2}$ Matthieu Wyart, ${ }^{3}$ and Francesco Zamponi $\oplus^{4}$ \\ ${ }^{1}$ Graduate School of Arts and Sciences, The University of Tokyo Tokyo 153-8902, Japan \\ ${ }^{2}$ Instituto de Física, UFRGS, 91501-970, Porto Alegre, Brazil \\ ${ }^{3}$ Institute of Physics, EPFL, CH-1015 Lausanne, Switzerland \\ ${ }^{4}$ Laboratoire de Physique de l'École Normale Supérieure, Université PSL, \\ CNRS, Sorbonne Université, Université de Paris, 75005 Paris, France
}

(Received 9 February 2020; accepted 4 May 2020; published 18 May 2020)

\begin{abstract}
We introduce a new model to study the effect of surface roughness on the jamming transition. By performing numerical simulations, we show that for a smooth surface, the jamming transition density and the contact number at the transition point both increase upon increasing asphericity, as for ellipsoids and spherocylinders. Conversely, for a rough surface, both quantities decrease, in quantitative agreement with the behavior of frictional particles. Furthermore, in the limit corresponding to the Coulomb friction law, the model satisfies a generalized isostaticity criterion proposed in previous studies. We introduce a counting argument that justifies this criterion and interprets it geometrically. Finally, we propose a simple theory to predict the contact number at finite friction from the knowledge of the force distribution in the infinite friction limit.
\end{abstract}

DOI: 10.1103/PhysRevLett.124.208001

Introduction.-Upon compression, a granular material suddenly acquires a finite mechanical pressure at a certain jamming transition density $\varphi_{J}$ at which constituent particles start to touch each other [1-9]. One of the most popular and simplest models of the jamming transition is a system consisting of frictionless spherical particles interacting via a purely repulsive potential. A notable property of the model is the so-called isostaticity: the number of degrees of freedom equals the number of constraints imposed by the contacts among constituent particles. A simple counting argument predicts that when a frictionless spherical system is isostatic, the contact number per particle is $z=2 d$ in $d$ spatial dimensions. Experiments [4] and numerical simulations $[10,11]$ show that the contact number at $\varphi_{J}$ indeed satisfies $z_{J}=2 d$. Remarkably, recent numerical and theoretical progress unveiled that isostatic systems, which encompass some classes of neural networks $[8,9,12,13]$ in addition to frictionless spherical particles, belong to the same universality class [14-18].

However, in experiments, friction has a significant effect on the jamming transition. Systematic numerical studies have been performed for spherical particles with the famous Mohr-Coulomb law: the tangential force $f_{t}$ between two particles in contact is proportional to the displacement from the point of contact as long as $\left|f_{t}\right| \leq \mu f_{n}$, where $f_{n}$ denotes the normal force and $\mu$ denotes the friction coefficient [19]. When the tangential force reaches the Coulomb threshold $\left|f_{t}\right|=\mu f_{n}$, the contact breaks and the particles start to slip with respect to each other. If we assume that each contact constrains one translational motion and $d-1$ tangential motions, the counting argument predicts $z_{J}=d+1$ when the system is isostatic $[1,20]$. However, numerical simulations show that $z_{J}$ smoothly decreases from $2 d$ upon increasing $\mu$, and converges to $d+1$ only in the large friction limit $\mu \rightarrow \infty[21,22]$. Isostaticity thus seems to be broken for frictional particles. However, more recently, it has been realized that more careful considerations are necessary to derive the isostatic condition for frictional particles [23-25]. The point is that a finite fraction of the fully mobilized contacts satisfy the Coulomb threshold $\left|f_{t}\right|=\mu f_{n}$, and those contacts do not constrain the tangential motion. This observation leads to a generalized isostaticity condition $z_{J}=d+1+2 n_{m} / d$, where $n_{m}$ denotes the number of fully mobilized contacts per particle [25]. Numerical simulations prove that frictional particles indeed satisfy generalized isostaticity at $\varphi_{J}$ when slowly equilibrated $[24,25]$.

Compared to frictionless particles, studies of the jamming of frictional particles, e.g., to unveil the mechanisms yielding the generalized isostaticity condition and their universality class, are still in their infancy. A reason is the strong nonanalyticity of the Coulomb law at the Coulomb threshold $\left|f_{t}\right|=\mu f_{n}$, which makes the contact network ill defined [25], and the lack of a well-defined potential energy [26]. A way to avoid this difficulty is to revisit the microscopic origin of the empirical Coulomb friction law. Although there are several possible origins of friction [27], here we focus on the geometric friction caused by surface roughness, which has gained a lot of attention due to the recent development of experimental techniques such as $3 \mathrm{D}$ printing $[28,29]$, and advanced computational techniques for complex-shaped particles $[30,31]$. In this 
work, we construct a new model to take into account the effect of surface roughness by means of a perturbative expansion around the reference case of spherical disks. By performing numerical simulations, we show that, for a smooth surface, $z_{J}$ of the model increases upon increasing asphericity, suggesting that a small deviation from perfect disks plays a similar role to the asphericity of convexshaped particles [5,32-38]. Contrarily, for a rough surface, $z_{J}$ decreases upon increasing asphericity, as for frictional particles. Furthermore, we show that our model gives a clear explanation for why particles with Coulomb friction satisfy the generalized isostaticity condition. Finally, we propose a simple approximation scheme to calculate $z_{J}$ for frictional particles.

Model.-We consider two-dimensional particles interacting with the repulsive harmonic potential [10]:

$$
V_{N}=\sum_{i<j}^{1, N} v\left(h_{i j}\left(\boldsymbol{x}_{i}, \boldsymbol{x}_{j}, u_{i}, u_{j}\right)\right), \quad v(h)=\frac{h^{2}}{2} \Theta(-h),
$$

where $\Theta(x)$ denotes the Heaviside step function, $\boldsymbol{x}_{i}=$ $\left\{x_{i}, y_{i}\right\}$ and $u_{i}$ denote the position and angle of the $i$ th particle, respectively, and $h_{i j}$ denotes the overlap function, which represents the minimal distance between particles $i$ and $j$. When particles $i$ and $j$ are overlapped, $h_{i j} \leq 0$, and otherwise, $h_{i j}>0$. The calculation of $h_{i j}$ is a nontrivial task for general shapes of nonspherical particles. To simplify the treatment, we assume that the shape of the particles is close to a disk. By means of a perturbation expansion around the reference disks, we obtain [36,38]

$$
\begin{aligned}
h_{i j}=\left|\boldsymbol{h}_{i j}\right| & =\left|\boldsymbol{h}_{i j}^{0}\right|+\delta \boldsymbol{h}_{i j} \cdot \frac{\boldsymbol{x}_{i}-\boldsymbol{x}_{j}}{\left|\boldsymbol{x}_{i}-\boldsymbol{x}_{j}\right|}+O\left(\delta \boldsymbol{h}_{i j}^{2}\right) \\
& \approx\left|\boldsymbol{x}_{i}-\boldsymbol{x}_{j}\right|-R_{i}-R_{j}+F\left(\boldsymbol{x}_{i}, \boldsymbol{x}_{j}, u_{i}, u_{j}\right),
\end{aligned}
$$

where $\boldsymbol{h}_{i j}$ and $\boldsymbol{h}_{i j}^{0}$, respectively, denote the vectors connecting the minimal paths between the surfaces of two particles and reference disks (Fig. 1), $\delta \boldsymbol{h}_{i j}=\boldsymbol{h}_{i j}-\boldsymbol{h}_{i j}^{0}$ denotes the deviation of the minimal path from the disks,

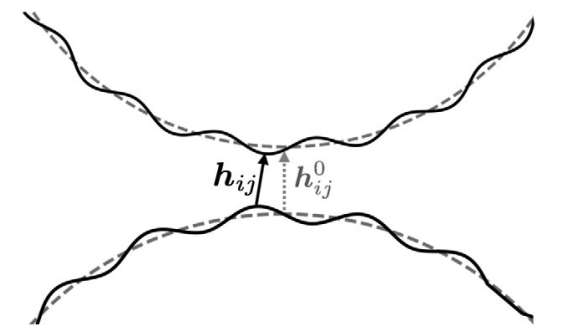

FIG. 1. Schematic picture of the surfaces of two particles. The solid lines represent the surfaces of particles, while the dashed lines represent the surfaces of the references disks. The solid and dashed arrows represent the minimal paths connecting the surfaces of particles and reference disks, respectively.
$R_{i}$ denotes the radius of the particles $i$, and we have introduced the auxiliary function $F \equiv \delta \boldsymbol{h}_{i j} \cdot\left(\boldsymbol{x}_{i}-\boldsymbol{x}_{j}\right) /$ $\left|\boldsymbol{x}_{i}-\boldsymbol{x}_{j}\right|$. To express the surface roughness, we require that $F$ is invariant under the following transformations: (i) the rotation without slip $u_{i} \rightarrow u_{i}+\delta / R_{i}$ and $u_{j} \rightarrow u_{j}-\delta / R_{j}$, where $\delta$ denotes an arbitrary constant with a dimension of length, and (ii) the global rotation. A functional form satisfying the above conditions is [39]

$$
\begin{aligned}
F\left(\boldsymbol{x}_{i}, \boldsymbol{x}_{j}, u_{i}, u_{j}\right) & =\left(R_{i}+R_{j}\right) f\left(\omega_{i j}\right), \\
\omega_{i j} & =\frac{R_{i} u_{i}+R_{j} u_{j}}{R_{i}+R_{j}}-\theta_{i j},
\end{aligned}
$$

where $\theta_{i j}$ denotes the angle between the relative vector $\boldsymbol{x}_{i}-\boldsymbol{x}_{j}$ and positive $x$ axis, namely, $\theta_{i j}=\operatorname{atan} 2\left(y_{j}-y_{i}\right.$, $\left.x_{j}-x_{i}\right)$. Although $f(\theta)$ can be any periodic function of period $\pi$, to make the connection with the Coulomb friction law, we consider the following specific form:

$$
f(\theta)=\mu \frac{2 \pi}{n} \operatorname{Tri}_{\varepsilon}\left(\frac{n}{2 \pi} \theta\right),
$$

where $n$ denotes an even number, and we have introduced a smoothed triangle wave function:

$$
\operatorname{Tri}_{\varepsilon}(x)= \begin{cases}-\frac{x^{2}}{2 \varepsilon}+\frac{1}{4}-\frac{\varepsilon}{2} & x \in[0, \varepsilon) \\ -x+\frac{1}{4} & x \in[\varepsilon, 1 / 2-\varepsilon) \\ \frac{(x-1 / 2)^{2}}{2 \varepsilon}-\frac{1}{4}+\frac{\varepsilon}{2} & x \in[1 / 2-\varepsilon, 1 / 2+\varepsilon) \\ x-\frac{3}{4} & x \in[1 / 2+\varepsilon, 1-\varepsilon) \\ -\frac{(x-1)^{2}}{2 \varepsilon}+\frac{1}{4}-\frac{\varepsilon}{2} & x \in[1-\varepsilon, 1),\end{cases}
$$

and $\operatorname{Tri}_{\varepsilon}(x \pm 1)=\operatorname{Tri}_{\varepsilon}(x)$. We show the typical behavior of $f(\theta)$ in Figs. 2(a) and 2(b). $f(\theta)$ depends on three parameters: $n, \mu$, and $\varepsilon$. Upon increasing $n$, the number of minima of $f(\theta)$ increases. Although solely $f(\theta)$ is not enough to determine the precise shape of particles, it is clear that the number of minima on the surface of a particle also increases with $n$, as schematically shown in Fig 2(c), suggesting that $n$ controls the roughness. $\mu / n$ represents the deviation from the reference disks, and our perturbative approach is justified only for $\mu / n \ll 1$. To make the physical meaning of $\mu$ clearer, we calculate the ratio between the normal and tangential forces between two particles in contact:

$$
\left|\frac{f_{t}}{f_{n}}\right|=\frac{R_{i}+R_{j}}{\left|\boldsymbol{x}_{i}-\boldsymbol{x}_{j}\right|}\left|f^{\prime}\left(\omega_{i j}\right)\right|,
$$

where $f_{n}=\partial_{x_{n}} v\left(h_{i j}\right)$ and $f_{t}=\partial_{x_{t}} v\left(h_{i j}\right) . \partial_{x_{n}}$ and $\partial_{x_{t}}$, respectively, denote the derivatives along the parallel and orthogonal directions to $\boldsymbol{x}_{i}-\boldsymbol{x}_{j}$. For $n \gg 1$ and at $\varphi_{J}$, $\left|\boldsymbol{x}_{i}-\boldsymbol{x}_{j}\right| \approx R_{i}+R_{j}$, and we get $\left|f_{t} / f_{t}\right| \approx f^{\prime}\left(\omega_{i j}\right) \leq \mu$, 
(a) $\quad-n=2-n=4-n=8$

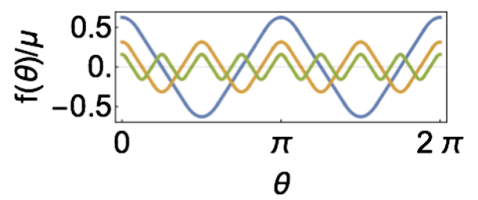

(b) $-\epsilon=0.25-\epsilon=0.1-\epsilon=0.0$

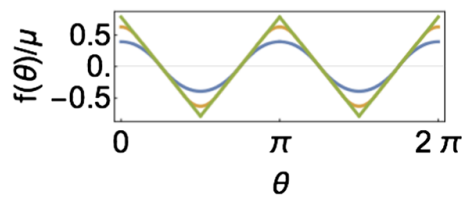

(c)

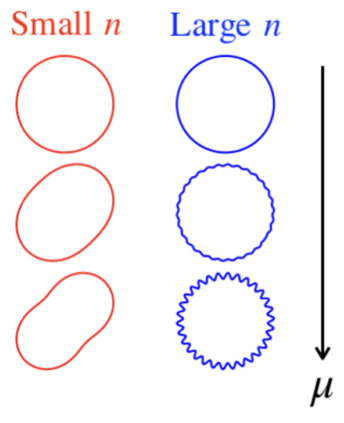

FIG. 2. (a) $f(\theta) / \mu$ for $\varepsilon=0.1$ and several $n$. The number of minima increases with $n$. (b) $f(\theta)$ for $n=2$ and several $\varepsilon$. The triangle wave is recovered when $\varepsilon=0$. (c) Schematic pictures of a particle shape. The shape deviates from the reference disk with $\mu$, and the surface becomes rougher for larger $n$.

implying that $\mu$ represents the effective friction coefficient. One may thus expect that the behavior of frictional particles can be recovered in the limit of a rough surface, $n \rightarrow \infty$ with fixed $\mu$. However, taking this limit is not enough because for $\varepsilon>0,\left|f_{t} / f_{n}\right|=f^{\prime}\left(\omega_{i j}\right)$ varies depending on $\omega_{i j}$ even when slip sets in, while $\left|f_{t} / f_{n}\right|=\mu$ for the Coulomb friction law [19]. The Coulomb friction law corresponds to the double limit $n \rightarrow \infty$ and $\varepsilon \rightarrow 0$, where $\left|f_{t} / f_{n}\right|<\mu$ if $\omega_{i j}$ is trapped in a minimum of $f\left(\omega_{i j}\right)$, and $\left|f_{t} / f_{n}\right|=\mu$ if slip sets in and $\omega_{i j} \in[\varepsilon, 1 / 2-\varepsilon) \cup[1 / 2+\varepsilon$, $1-\varepsilon)$.

Numerics. - We perform numerical simulations for $N=$ 128 particles consisting of the same number of large and small particles under periodic boundary conditions. The radii of small and large particles are $R_{S}=0.5$ and $R_{L}=0.7$, respectively. We find $\varphi_{J}$ by combining slow isotropic compression and decompression as follows [10]. We first generate a random initial configuration at a small packing fraction $\varphi=0.1$. Then, we slowly compress the system. For each compression step, we increase the packing fraction with a small increment $\delta \varphi=10^{-4}$, and successively minimize the energy with the FIRE algorithm [40] until the squared force acting on each particle becomes smaller than $10^{-25}$. After arriving at a jammed configuration with $V_{N} / N>10^{-16}$, we change the sign and amplitude of the increment as $\delta \varphi \rightarrow-\delta \varphi / 2$. Then, we decompress the system until we obtain an unjammed configuration with $V_{N} / N<10^{-16}$. We repeat this process by changing the sign and amplitude of the increment as $\delta \varphi \rightarrow-\delta \varphi / 2$ every time the system crosses $\varphi_{J}$. We terminate the simulation when $V_{N} / N \in\left(10^{-16}\right.$, $\left.2 \times 10^{-16}\right)$. Then, we remove the rattlers that have zero or one contact, and calculate the physical quantities. To improve the statistics, we average over 10 independent samples. (a)
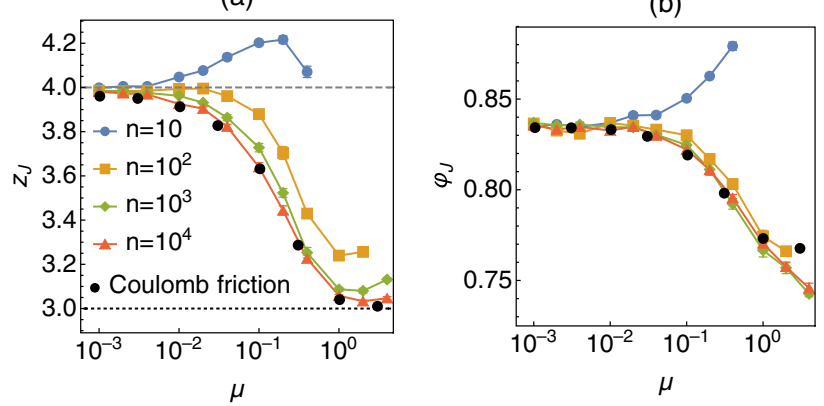

FIG. 3. $\mu$ dependence for fixed $\varepsilon=0.1$ of (a) the contact number per particle at the jamming transition point $z_{J}$ and (b) the jamming transition point $\varphi_{J}$. The data for the Coulomb friction model were taken from Ref. [42].

Results.-First, we discuss the behavior for $\varepsilon=0.1$. In Fig. 3(a), we show the contact number per particle at the jamming transition point $z_{J}$. For small $n, z_{J}$ increases upon increasing $\mu$; see the data for $n=10$. Since $\mu / n$ represents the deviation from disks, this behavior is qualitatively similar to that observed in convex-shaped particles $[5,32-$ 38]. Contrarily, for large $n, z_{J}$ decreases with $\mu$ [41]. For the largest value of $n, n=10^{4}, z_{J}$ quantitatively agrees with previous results generated by isotropic compression of frictional particles for the same system size, $N=128$ [42]. In Fig. 3(b), we show the jamming transition point $\varphi_{J}$. As for $z_{J}, \varphi_{J}$ increases with $\mu$ for small $n$, and decreases for large $n$. For $n=10^{4}$ and $\mu \lesssim 1$, the behavior of $\varphi_{J}$ is similar to that of the Coulomb friction model, while for $\mu \gtrsim 1$, there is a small but visible deviation. We guess that this discrepancy for large $\mu$ is due to the difference in the algorithms used for the minimization: for our model, the energy was minimized by the FIRE algorithm, while for frictional particles, the kinetic energy was minimized by molecular dynamics simulation with a damping proportional to the force [42]. Further studies are necessary to clarify this point. To see the $\varepsilon$ dependence, in Fig. 4 we (a)

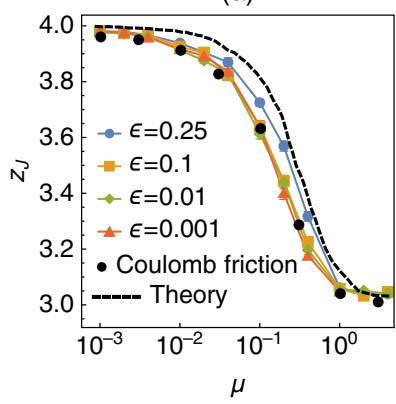

(b)

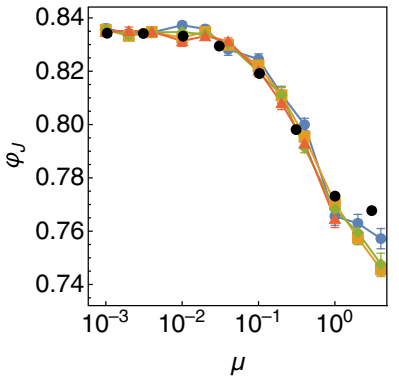

FIG. 4. $\mu$ dependence for fixed $n=10^{4}$ of (a) the contact number per particle at the jamming transition point $z_{J}$ and (b) the jamming transition point $\varphi_{J}$. Markers denote the numerical results, while the dashed line denotes the theoretical prediction; see main text. 
(a)

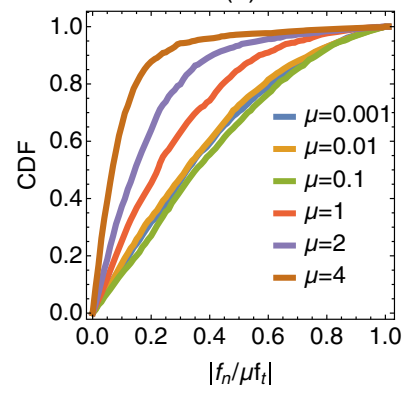

(b)

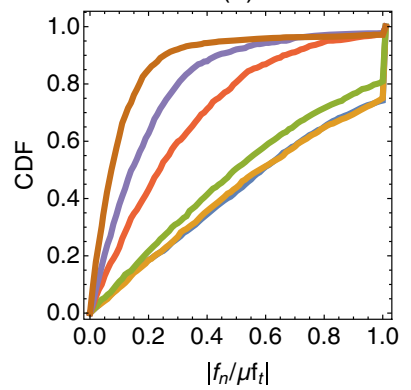

(c)

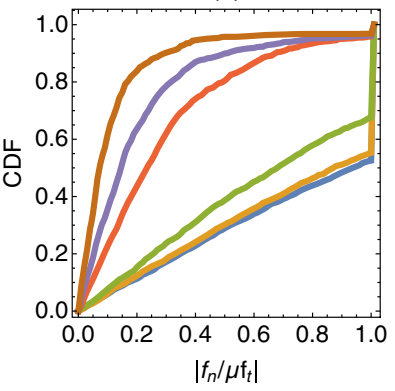

(d)

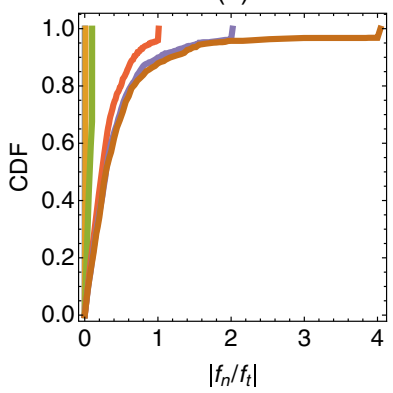

FIG. 5. (a)-(c) Cumulative distributions of $\left|f_{n} / \mu f_{t}\right|$ for $n=10^{4}$ and $\varepsilon=0.25,0.1$, and 0.01 , respectively. (d) Cumulative distribution of $\left|f_{n} / f_{t}\right|$ for $n=10^{4}$ and $\varepsilon=0.01$.

show $z_{J}$ and $\varphi_{J}$ for $n=10^{4}$ and several $\varepsilon . z_{J}$ and $\varphi_{J}$ do not exhibit a significant $\varepsilon$ dependence and agree well with the results for the Coulomb friction law.

In Figs. 5(a)-5(c), we show the cumulative distribution function (CDF) of $\left|f_{n} / \mu f_{t}\right|$ for $n=10^{4}$ and several $\varepsilon$. For $\varepsilon=0.25$, the CDF smoothly increases with $\left|f_{n} / \mu f_{t}\right|$. Contrarily, for $\varepsilon<0.25$, the CDF has a singular peak at $\left|f_{n} / \mu f_{t}\right|=1$. The peak grows upon decreasing $\mu$ and $\varepsilon$. In Fig. 5(d), we show the CDF of $\left|f_{n} / f_{t}\right|$ for $n=10^{4}$ and $\varepsilon=0.01$. The CDF converges to a constant distribution for large $\mu$.

The strong peak of the CDF at $\left|f_{t} / \mu f_{n}\right|=1$ indicates that there are a finite fraction of contacts satisfying the Coulomb threshold $\left|f_{t}\right|=\mu f_{n}$. Those contacts are referred to as the fully mobilized contacts [1]. As the fully mobilized contacts do not constrain the tangential motion, the total number of constraints imposed by the contacts is $N z-N n_{m}$, where $N n_{m}$ denotes the number of fully mobilized contacts. This should be equated to the number of degrees of freedom $3 N$ when the system is isostatic. Therefore, for an isostatic system, $n_{m}$ is [25]

$$
n_{m}=z-3 \text {. }
$$

In Fig. 6, we test this conjecture for $n=10^{4}$. The plot clearly shows that the numerical data converge to the

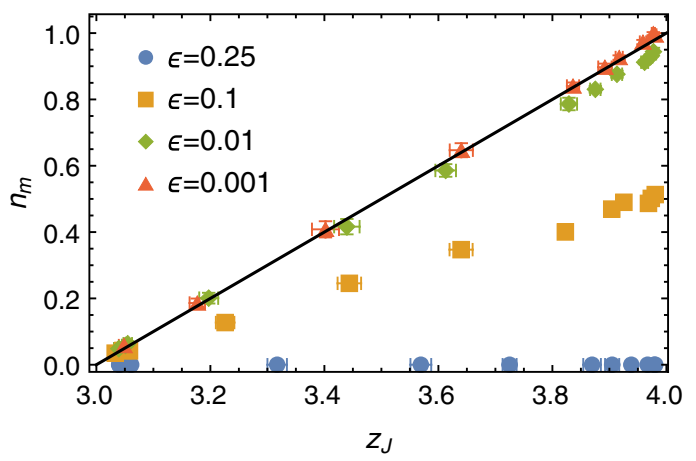

FIG. 6. Relation between $n_{m}$ and $z_{J}$ for $n=10^{4}$. Markers denote numerical results. The solid line denotes the theoretical prediction $n_{m}=z-3$ corresponding to generalized isostaticity. theoretical prediction, Eq. (7), in the Coulomb friction limit $\varepsilon \rightarrow 0$.

Theory.-Here we show that the generalized isostaticity in the $\varepsilon \rightarrow 0$ limit can be explained by a simple counting argument, which slightly generalizes the corresponding one for nonspherical particles in two dimensions [1]. At $\varphi_{J}$, $h_{i j}=0$ for all contacts. This can be satisfied when the number of degrees of freedom $3 N$ is larger than the number of contacts $N z_{J} / 2$ :

$$
3 N \geq \frac{N z_{J}}{2} \rightarrow z_{J} \leq 6
$$

Additionally, a stable system should satisfy the $3 N$ force balance equations:

$$
\frac{\partial V_{N}}{\partial x_{i}}=0, \quad \frac{\partial V_{N}}{\partial y_{i}}=0, \quad \frac{\partial V_{N}}{\partial u_{i}}=0 .
$$

Those are linear combinations of the normal and tangential forces, $f_{n}$ and $f_{t}$, between particles in contact. Considering that there are $N n_{m}$ fully mobilized contacts, the degree of freedom of $f_{n}$ and $f_{t}$ is $N z_{J}-N n_{m}$. Therefore, Eqs. (9) have nontrivial solutions only if $[24,25]$

$$
3 N \leq N z_{J}-N n_{m} \rightarrow z_{J} \geq 3+n_{m} .
$$

This inequality generally holds for any $\varepsilon$; see Fig. 6 . From Eqs. (8) and (10), at $\varphi_{J}$, we have

$$
3+n_{m} \leq z_{J} \leq 6,
$$

implying that the generalized isostaticity does not hold in general.

We can improve the counting argument in the $\varepsilon \rightarrow 0$ limit, where $\omega_{i j}$ of nonmobilized contacts should be located precisely at a minimum of $f\left(\omega_{i j}\right)$ because the corresponding stiffness diverges as $f^{\prime \prime}\left(\omega_{i j}\right) \sim \varepsilon^{-1}$. This provides $N z_{J} / 2-N n_{m}$ additional constraints. Thus, Eq. (8) should be modified as

$$
3 N \geq N z_{J}-N n_{m} \rightarrow z_{J} \leq 3+n_{m} .
$$


Equations (10) and (12) prove the generalized isostaticity Eq. (7). It is not straightforward to generalize the above argument to higher dimensions. We left it as future work.

A theoretical challenge is to predict the $\mu$ dependence of $z_{J}$. In Fig. 4(a), we show that $z_{J}$ does not show a strong $\varepsilon$ dependence. This allows us to focus on small $\varepsilon$, e.g., $\varepsilon=0.01$, where the generalized isostaticity, Eq. (7), may simplify the treatment, as it directly connects $z_{J}$ to $n_{m}$. We tentatively approximate $n_{m}$ by neglecting the $\mu$ dependence of the distribution of $\left|f_{t} / f_{n}\right|$ and calculate $n_{m}$ as

$$
n_{m} \approx \int_{\mu}^{\infty} d x P_{\infty}(x)=1-\operatorname{CDF}\left(\left|f_{t} / f_{n}\right|=\mu\right),
$$

where $P_{\infty}(x)$ denotes the distribution of $\left|f_{t} / f_{n}\right|$ in the limit $\mu \rightarrow \infty$. As shown in Fig. 5(d), the CDF of $\left|f_{t} / f_{n}\right|$ for $\varepsilon=0.01$ is converged to a constant distribution for $\mu \gtrsim 2$. So, we use the CDF for $\mu=4$. In Fig. 4(a), we show our theoretical prediction $z_{J} \approx n_{m}+3 \approx 4-\operatorname{CDF}\left(\left|f_{t} / f_{n}\right|=\right.$ $\mu$ ) with the black dashed line. The agreement is not perfect but still surprisingly nice, considering the simplicity of the theory and the fact that there are only a few theories for the jamming of frictional particles $[43,44]$.

Conclusions. - We constructed a model that takes into account the effect of surface roughness by means of a perturbation expansion around ideal disks. By changing the surface roughness, the model can smoothly interpolate the phenomenology of frictionless convex-shaped particles and frictional disks.

We found that the fraction of fully mobilized contacts strongly depends on $\varepsilon$, and, consequently, the generalized isostaticity condition is satisfied only in the limit of the Coulomb friction law, $\varepsilon \rightarrow 0$. However, our investigation is limited to a specific class of functions $f\left(\omega_{i j}\right)$ described by Eq. (4), and we also assumed that two particles have at most one contact and neglected the effect of multiple contacts. It would be desirable to investigate a broader class of $f\left(\omega_{i j}\right)$, allowing multiple contacts, to clarify under which conditions the system satisfies generalized isostaticity.

We warmly thank J.-P. Bouchaud for discussions related to this work. This project has received funding from the European Research Council (ERC) under the European Union's Horizon 2020 research and innovation program (Grant Agreement No. 723955-GlassUniversality).

*hikeda@g.ecc.u-tokyo.ac.jp

[1] M. Van Hecke, J. Phys. Condens. Matter 22, 033101 (2010).

[2] D. J. Durian, Phys. Rev. Lett. 75, 4780 (1995).

[3] Z. Zhang, N. Xu, D. T. Chen, P. Yunker, A. M. Alsayed, K. B. Aptowicz, P. Habdas, A. J. Liu, S. R. Nagel, and A. G. Yodh, Nature (London) 459, 230 (2009).

[4] J. Bernal and J. Mason, Nature (London) 188, 910 (1960).

[5] A. Donev, I. Cisse, D. Sachs, E. A. Variano, F. H. Stillinger, R. Connelly, S. Torquato, and P. M. Chaikin, Science 303, 990 (2004).
[6] A. Jaoshvili, A. Esakia, M. Porrati, and P. M. Chaikin, Phys. Rev. Lett. 104, 185501 (2010).

[7] D. Bi, J. Lopez, J. Schwarz, and M. L. Manning, Nat. Phys. 11, 1074 (2015).

[8] S. Franz and G. Parisi, J. Phys. A 49, 145001 (2016).

[9] S. Franz, S. Hwang, and P. Urbani, Phys. Rev. Lett. 123, 160602 (2019).

[10] C. S. O'Hern, L. E. Silbert, A. J. Liu, and S. R. Nagel, Phys. Rev. E 68, 011306 (2003).

[11] C. P. Goodrich, A. J. Liu, and S. R. Nagel, Phys. Rev. Lett. 109, 095704 (2012).

[12] S. Franz, G. Parisi, M. Sevelev, P. Urbani, and F. Zamponi, Sci. Post Phys. 2, 019 (2017).

[13] S. Franz, A. Sclocchi, and P. Urbani, Phys. Rev. Lett. 123, 115702 (2019).

[14] M. Wyart, Ann. Phys. (Paris) 30, 1 (2005).

[15] M. Wyart, L. E. Silbert, S. R. Nagel, and T. A. Witten, Phys. Rev. E 72, 051306 (2005).

[16] E. DeGiuli, E. Lerner, C. Brito, and M. Wyart, Proc. Natl. Acad. Sci. U.S. A. 111, 17054 (2014).

[17] P. Charbonneau, J. Kurchan, G. Parisi, P. Urbani, and F. Zamponi, Nat. Commun. 5, 3725 (2014).

[18] P. Charbonneau, E. I. Corwin, G. Parisi, and F. Zamponi, Phys. Rev. Lett. 114, 125504 (2015).

[19] P. A. Cundall and O. D. Strack, Geotechnique 29, 47 (1979).

[20] S. F. Edwards and D. V. Grinev, Phys. Rev. Lett. 82, 5397 (1999).

[21] T. Unger, J. Kertész, and D. E. Wolf, Phys. Rev. Lett. 94, 178001 (2005).

[22] L. E. Silbert, Soft Matter 6, 2918 (2010).

[23] J.-P. Bouchaud, arXiv:cond-mat/0211196.

[24] K. Shundyak, M. van Hecke, and W. van Saarloos, Phys. Rev. E 75, 010301(R) (2007).

[25] S. Henkes, M. van Hecke, and W. van Saarloos, Europhys. Lett. 90, 14003 (2010).

[26] J. Chattoraj, O. Gendelman, M. P. Ciamarra, and I. Procaccia, Phys. Rev. Lett. 123, 098003 (2019).

[27] B. N. Persson, Sliding Friction: Physical Principles and Applications (Springer Science \& Business Media, Switzerland AG, 2013).

[28] A. G. Athanassiadis, M. Z. Miskin, P. Kaplan, N. Rodenberg, S. H. Lee, J. Merritt, E. Brown, J. Amend, H. Lipson, and H. M. Jaeger, Soft Matter 10, 48 (2014).

[29] L. C. Hsiao and S. Pradeep, Curr. Opin. Colloid Interface Sci. 43, 94 (2019).

[30] F. Alonso-Marroquin, Europhys. Lett. 83, 14001 (2008).

[31] S. Papanikolaou, C. S. O’Hern, and M. D. Shattuck, Phys. Rev. Lett. 110, 198002 (2013).

[32] A. Donev, R. Connelly, F. H. Stillinger, and S. Torquato, Phys. Rev. E 75, 051304 (2007).

[33] S. R. Williams and A. P. Philipse, Phys. Rev. E 67, 051301 (2003)

[34] J. Blouwolff and S. Fraden, Europhys. Lett. 76, 1095 (2006).

[35] K. VanderWerf, W. Jin, M. D. Shattuck, and C. S. O'Hern, Phys. Rev. E 97, 012909 (2018).

[36] C. Brito, H. Ikeda, P. Urbani, M. Wyart, and F. Zamponi, Proc. Natl. Acad. Sci. U.S.A. 115, 11736 (2018).

[37] H. Ikeda, P. Urbani, and F. Zamponi, J. Phys. A 52, 344001 (2019). 
[38] H. Ikeda, C. Brito, and M. Wyart, J. Stat. Mech. 2020, 033302 (2020).

[39] $F$ is not a periodic function of $u_{i}$ and $u_{j}$. But this is not a big problem because such symmetries do not affect the local stability arguments discussed here.

[40] E. Bitzek, P. Koskinen, F. Gähler, M. Moseler, and P. Gumbsch, Phys. Rev. Lett. 97, 170201 (2006).

[41] For large $n, z_{J}$ has a minimum at an intermediate value of $\mu$. This counterintuitive behavior could be a signature of the breakdown of our perturbative approach. The position of the minimum shifts to higher $\mu$ upon increasing of $n$, and the result of the Coulomb friction law is correctly reproduced in the limit of $n \rightarrow \infty$.

[42] F. Xiong, P. Wang, A. H. Clark, T. Bertrand, N. T. Ouellette, M. D. Shattuck, and C. S. O’Hern, Granular Matter 21, 109 (2019).

[43] C. Song, P. Wang, and H. A. Makse, Nature (London) 453, 629 (2008).

[44] T. Shen, S. Papanikolaou, C. S. O'Hern, and M. D. Shattuck, Phys. Rev. Lett. 113, 128302 (2014). 